\title{
JAK inhibitors: Evolving therapies in rheumatoid arthritis
}

\author{
Shiva Shankar Jha
}

Director \& Head of Dept. of Orthopaedics, Mahavir Vaatsalya Aspatal, Patna, Bihar, India

Corresponding Author

Email:drssjha@gmail.com

\begin{abstract}
In rheumatoid arthritis, there has been a paradigm shift from controlling symptoms to controlling the disease progress with abrogation of inflammation. Achieving this remission or very low disease activity has become increasingly possible with USA FDA approved first JAK inhibitor, Tofacitinib in November 2012 (not in Europe). This new small molecule JAK inhibitors are oral drug working intra-cellularly in comparison to TNF $\alpha$ inhibitors targeting a singly cytokine within extra-cellular space confining themselves to cell surface receptors. The second JAK inhibitor Baracitinib approved in Europe in February 2017 and in USA in May 2018 has greater promises. Once daily $2 \mathrm{mg}$ oral dose used either as monotherapy or in combination with methotrexate in moderate to severe active rheumatoid arthritis in adults have provided remission, even in patients who have responded inadequately or are intolerant to one or more DMARDs / bDMARDs. It is a safe second line therapy after methotrexate. In DMARD naïve patients, it is likely to become first-line agents
\end{abstract}

Keywords: JAK inhibitor, TNF $\alpha$ inhibitor, NSAID, bDMARD, Methotrexate, Tofacitinib.

Management of rheumatoid arthritis is still evolving. The evolving practice has encountered several approaches aimed at: comprehensive, standard or minimal tailoring to health systems, treating to target versus stepwise schedule, tighter goals, individualized treatment goals and new treatment. Apart from achieving these goals, new treatment options have become available reinforcing the management practices in rheumatoid arthritis. The management has travelled far and wide from the day of use of pain killers and NSAIDs to reversal of pyramid and use of conventional DMARDs, and finally to biologics and biomarkers. These biologics and biomarkers had many additions to the family with some limitations to their use including price. Steroids have been a common accompaniment. However, of late, remissions have become achievable in majority of these cases. Introduction of the newer drugs have the advantage of being delivered orally with many promises. All these treatment modalities have goals to relieve pain, reduce inflammation, slow joint damage and improve functioning.

\section{Modern Era of Treatment \\ 1980s \& 1990s: 1980s heralded the modern era of treatment. Methotrexate was still the corner stone of management and invariably in one third of patients, methotrexate alone could control the disease. 1990s saw the introduction of revolutionary narrow targeting cytokines and were preferred over general immuno- suppressants. The TNF $\alpha$ inhibitor targeting a single cytokine works within the extra-cellular space and targets cell surface receptors.}

\section{Past Decade}

In the past decade, treatment of rheumatoid arthritis got revolutionized and various biological DMARDs came into clinical practice. Various TNF inhibitors were in practice: infliximab, etanercept, adalimumab, golimumab and certolizumab. Simultaneously other biologicals with different mechanism of action came into the market: anti-CD 20 monoclonal antibody (Rituximab), IL-6 receptpr inhibitors (Tocilizumab), Tcell co-stimulation inhibitor (Abatacept).

Despite dramatic improvement with the biologicals, there were some unmet needs. Patients refractory to treatment, patients losing efficacy of the biologicals and patients not tolerating these biologics were the main obstacles in achieving remission of the disease in such situations.

In the present modern era of treatment, in spite of single cytokine targeting becoming effective, not every patient responded because many different cytokines were involved in the pathogenesis of rheumatoid arthritis. Hence, there was a challenge to develop alternatives to single cytokine inhibition.

\section{Goal of Therapy: Remission}

Goal of therapy is to achieve remission or very low disease activity status. Remission is defined as complete absence of clinical signs and symptoms of synovitis, elimination of silent synovial inflammation and normalization of markers of systemic inflammation such as ESR and CRP. This is a paradigm shift from controlling symptoms to controlling the disease progress with abrogation of inflammation. Achieving remission seems reachable in the modern era of management.

EULAR phase I recommendations were updated in 2016. On clinical diagnosis of RA, methotrexate ${ }^{1}$ could $^{2}$ be started if there are no contraindications for use of this drug. 


\section{6 updated EULAR recommedations: Phase I}

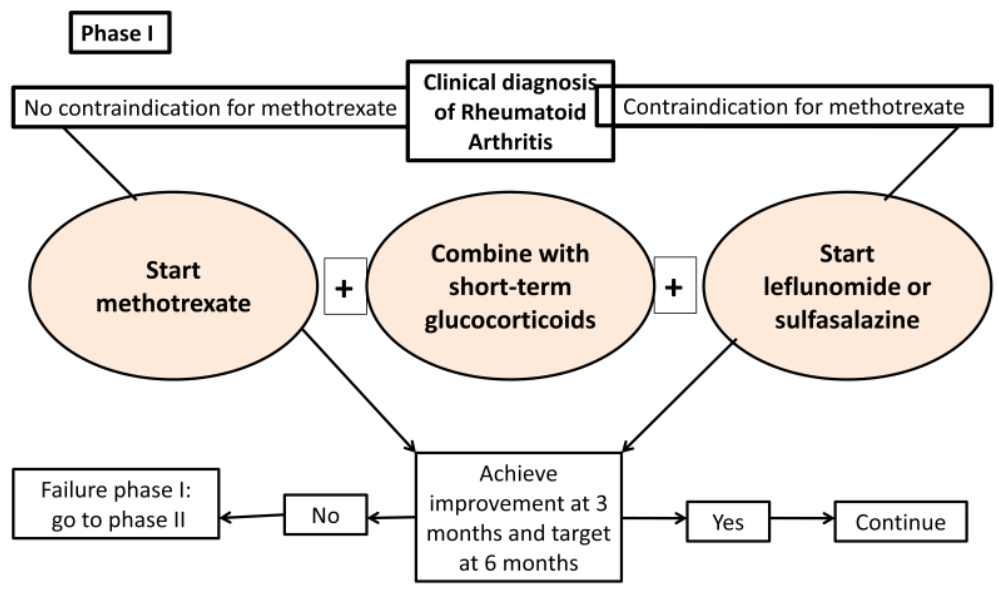

Leflunomide or sulfasalazine was started in patients with contraindication for methotrexate. A short course of glucocorticoids was recommended to be combined with either drug to achieve improvement at three months and achieve target at six months. On achieving this improvement and target, the drug was allowed to continue. Switch over to phase II EULAR recommendation (2016) was initiated on failure for lack of efficacy and / or toxicity in phase I to achieve the target within six months.

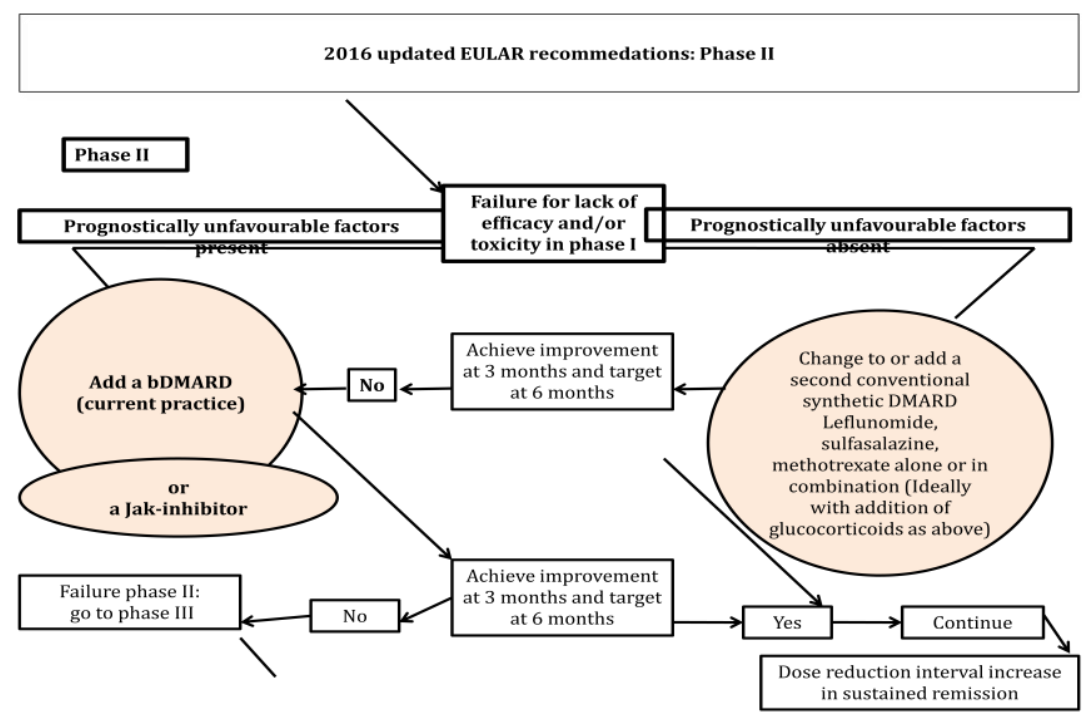

In presence of prognostically unfavourable factors, a biological DMARD (current practice) is added or a JAK inhibitor is started. In absence of prognostically unfavourable factors, change to or addition of a second conventional synthetic DMARD i.e. lefluonomide, sulfasalazine, methotrexate alone or in combination (ideally with addition of glucocorticoids), is recommended. Again on achieving improvement at 3 months and target at 6 months, this combination continues even after remission but the dose reduction interval increases in sustained remission. On failure to achieve the target, the patient is switched over to a bDMARD or a JAK-inhibitor. On failure or lack of efficacy or toxicity in phase II, the management is switched over to phase III EULAR recommendation (2016). 


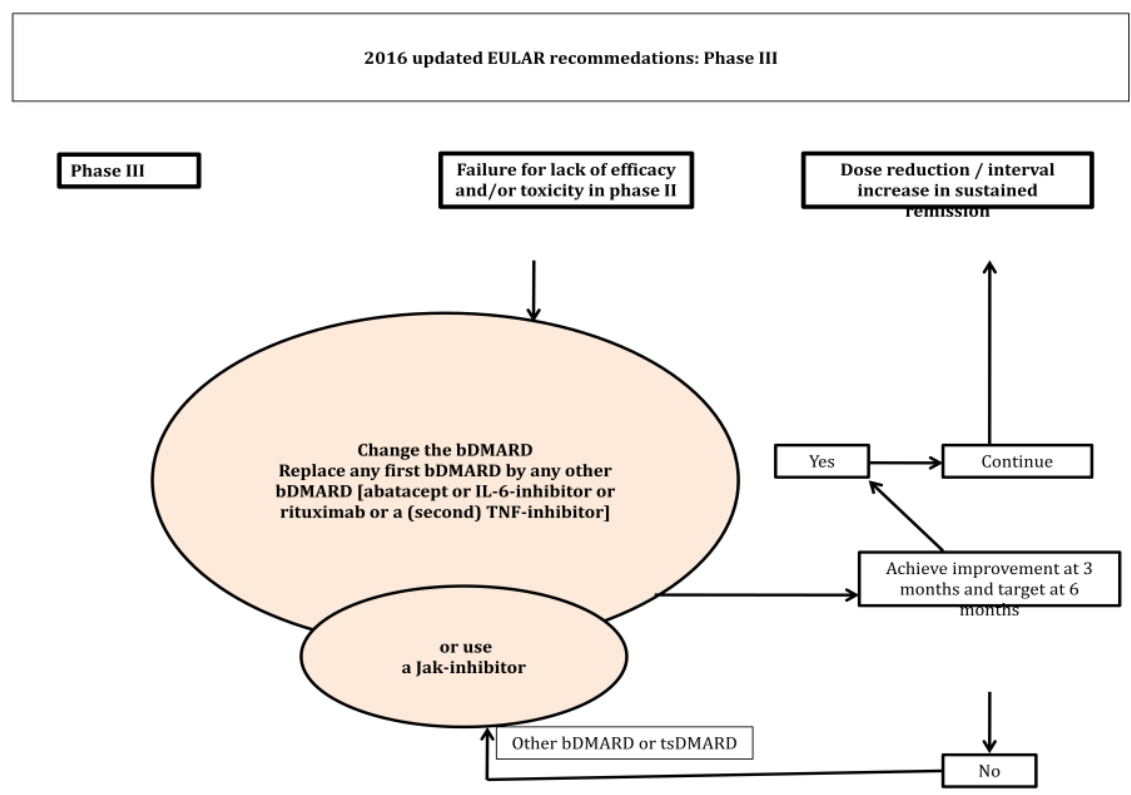

The previous bDMARD is changed and replaced by any other bDMARD (abatacept or IL-6-inhibitor or rituximab or a second TNF-inhibitor). Alternatively, straightaway, a JAK-inhibitor can be started either from the beginning of phase III or on failure of the bDMARD. On failure, the other option again is to substitute it with other bDMARD before changing to a JAK-inhibitor.

As is evident from these three updated EULAR recommendations (2016) from phase I, II and III, it is clear that use of JAK-inhibitor is recommended in phase II and III for the patients who have failed for lack of efficacy and / or toxicity of first bDMARD or even a switchover bDMARD.

\section{New Era of RA Treatment}

Heralding the transition to new era of rheumatoid arthritis treatment with small molecule has aroused hope for early and definite remission of the disease. First JAKinhibitor, the new small molecule, Tofacitinib oral drug was approved by USA FDA in November 2012 (not in Europe) only for the management of rheumatoid arthritis. $^{1}$

JAKs are Receptor Tyrosine Kinase family in mammals and are JAK1, JAK2, JAK3 and $\mathrm{TYK}^{3}$. Name comes from Roman God JANUS, a two faced god of beginning and transition looking at the past and the future.

\begin{tabular}{|l|}
\hline Face of God of Beginning \\
\hline Looking at the past \\
\hline
\end{tabular}
their 2 domains: JH1-domain at the Carboxyl end and $\mathrm{JH} 2$ - a Kinase-like domain lacking in catalytic activity but having important regulatory role. JAKs are attractive targets for treatment of autoimmune diseases. They mediate signaling via surface receptor for several proinflammatory cytokines involved in rheumatoid arthritis.

\section{Targeting cytokine signaling pathway}

Various cytokine signaling pathways are targeted using JAK inhibitors in various combinations. ${ }^{4}$ JAK 1 and JAK3 target cytokine receptors sharing the chain involving IL-2, 4, 7, 15 and 21. JAK 1, TYK2 and JAK2 target cytokine receptors sharing IL-4. JAK 2 targets homodimerics cytokine receptors GH, EpO an GM-CSF. JAK1, JAK2 and TYK2 target interferon (type E receptors) involving IFN. JAK2 and TYK2 target cytokine receptors sharing IL-12-R $\beta 1$ subunit involving IL-12 and IL-23. ${ }^{6}$

\section{Pathogenesis}

JAK inhibitors prevent signaling of JAK enzymes leading to interruption of signal transduction of cytokines. They are selective inhibitor of JAK1 and 
JAK3. JAK1 binds to $\beta$-subunit of cytokine receptor such as IL-2, 4, 7, 9, 15 and 21. It binds to common $Y_{c}$ chain of these IL. ${ }^{6}$

JAK3 are expressed in lymphoid cells and nervous system in hematopoietic tissues, myeloid cells, NK cells and activated $\mathrm{B}$ and $\mathrm{T}$ cells.

Autotransphosphorylation takes place on bondage of cytokines to receptor JAK-1 or 3 . Binding and activation of STAT proteins ${ }^{7}$ takes place. STAT proteins, can be translocated to nucleus which regulates transcription of several genes critical of immune response.

Tofacitinib is used as 5/10 mg twice daily. ${ }^{2}$ Used as monotherapy is efficatious in improving physical function in patients not tolerating methotrexate and most bDMARD. Tofacitinib is a targeted synthetic (ts) DMARD for oral use. The small molecule of this drug provide the benefit of targeting increased biological disease activity across multiple pathways by inhibiting intracellular signaling of cytokines and growth factors. It is interesting to note that chemically, it is related more to traditional methotrexate than to bDMARD. Action wise is like bDMARD in their suppression of cytokines. Onset of response occurs within two weeks and the efficacy is maintained up to five years.

\section{Safety Issues with Tofacitinib}

Safety issues are multi-fold: a. increased rate of infection-upper respiratory tract, urinary Tract, bronchitis, herpese zoster and few cases of tuberculosis, b. increased low density lipoprotein levels and aminotransferase, c. cytopenias-neutropenia, anemia and thrombocytopenia, d. small increase in creatinin levels and gastrointestinal adverse events. ${ }^{5}$

\section{Other Indications}

Tofacitinib can be used in other indications apart from rheumatoid arthritis: psoriasis, psoriatic arthritis, spondyloarthropathy, ulcerative colitis, lupus and Chron's disease.

New JAk-SyK Inhibitors, Baracitinib, the second JAK inhibitor was approved in Europe in February 2017 and by US FDA in May 2018. It is now the mainstay of $J A K$ inhibitor therapy. It is used as oral once daily in 2 $\mathrm{mg}$ (rarely $4 \mathrm{mg}$ ) dose. It is reversibile inhibitor for JAK1 and 2. It is used as either monotherapy or in combination with methotrexate for treatment of moderate to severe active rheumatoid arthritis in adults, who have responded inadequately or who are intolerant to one or more DMARDs.

In placebo-controlled trials, Baricitinib slowed disease progression and reduced disease activity, symptoms and disability in patients previously treated with conventional and / or bDMARDs.

Before initiating or during therapy with Baricitinib (Tofacitinib earlier), vaccinations are recommended: pneumococcal, influenza and hepatitis B (killed vaccine), human papilloma (recombinant vaccine) and herpes zoster (live vaccine). Prophylactic immunization with live zoster vaccine should not be given while on treatment with bDMARD.

\section{Newer Treatments}

With deeper understanding of pathophysiological mechanisms of rheumatoid arthritis, there has been an increasing interest in assessing potential efficacy of novel agents targeting a cytokine IL-17 during recent years. The two drugs Secukinumab (fully human anti-IL-17a antibody) and Ixekizumab (humanized IgG4 mAb against IL-17) belong to this class and claims to have improved signs and symptoms of rheumatoid arthritis with no strong adverse safety signal when added to DMARDs.

\section{Personalized Treatment}

With rapidly growing number of available potent agents, there has been obvious need for a more personalized treatment to limit long-term consequences. Guidelines have moved towards earlier and more aggressive treatment of rheumatoid arthritis. JAK inhibitors are changing rheumatoid arthritis treatment paradigm. It is a safe second line therapy after methotrexate. In DMARD naïve patients, it is likely to become first-line agents, because it is more effective than methotrexate. Long-term safety can be achieved with monitoring and vaccination. The unique mechanism of action with rapid onset of clinical response and efficacy, looks promising. Cost is a definite challenge.

Funding: No funding sources.

Conflict of interest: None declared.

\section{References}

1. https://ard.bmj.com/content/76/6/960

2. https://reference.medscape.com/drug/xeljanz-xeljanz-xrtofacitinib-999789

3. Marc D. Cohen Edward C. Keystone. JAK Inhibitors for Rheumatology Arthritis. Curr Treat Options in Rheum 2015;1:305-19.

4. Kontzias A, et al. Curr Opin Pharmacol 2012;12:464-70.

5. https://www.accessdata.fda.gov/drugsatfda_docs/label/201 8/203214s01lbl.pdf

6. Leonard $\mathrm{H}$. et al; New horizons in the treatment and management of rheumatoid arthritis: How Janus Kinase (JAK) Inhibitors are changing the treatment Paradigm. Journal of Managed Care Medicine; 2017;20(2)Supplement.

7. Winthrop K L. Nat Rev Rheumatol 2017;13:234-43.

How to cite this article: Jha S. JAK inhibitors: Evolving therapies in rheumatoid arthritis. IP Int $J$ Orthop Rheumatol 2018;4(2):43-6. 